$$
\text { Coif- } 950226--32
$$

UCRL-JC-117560

PREPRINT

\title{
Three Dimensional Imaging of DNA Fragments During Electrophoresis Using a Confocal Detector
}

\author{
Laurence R. Brewer \\ Courtney Davidson \\ Joe Balch \\ Anthony Carrano
}

This paper was prepared for submittal to

the Society of Photo-Optical Instrumentation Engineers

San Jose, CA

February 8, 1995

January 30, 1995

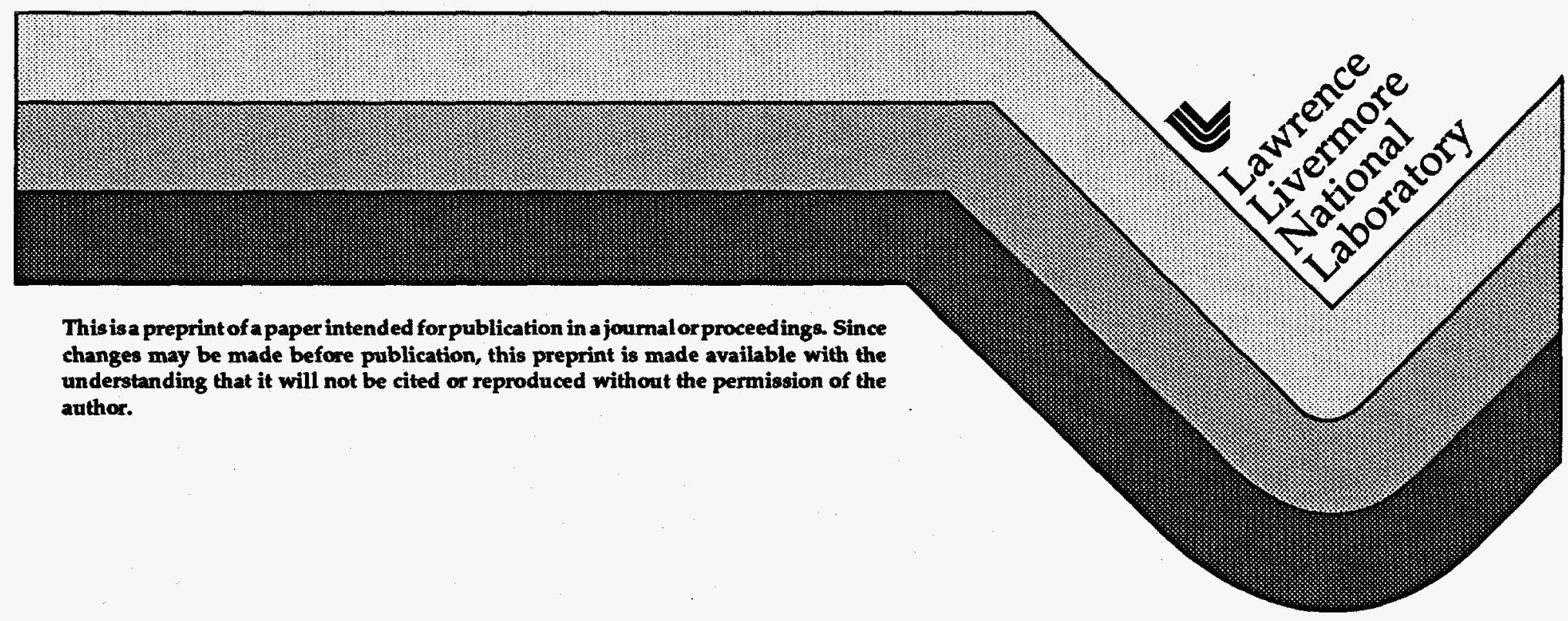

This is a preprint of a paper intended for publication in a journal or proceedings. Since changes may be made before publication, this preprint is made available with the understanding that it will not be cited or reproduced without the permission of the author. 


\section{DISCLAIMER}

This document was prepared as an account of work sponsored by an agency of the United States Government. Neither the United States Government nor the University of California nor any of their employees, makes any warranty, express or implied, or assumes any legal liability or responsibility for the accuracy, completeness, or usefulness of any information, apparatus, product, or process disclosed, or represents that its use would not infringe privately owned rights. Reference herein to any specific commercial product, process, or service by trade name, trademark, manufacturer, or otherwise, does not necessarily constitute or imply its endorsement, recommendation, or favoring by the United States Government or the University of California. The views and opinions of authors expressed herein do not necessarily state or reflect those of the United States Government or the University of California, and shall not be used for advertising or product endorsement purposes. 


\section{DISCLAIMER}

Portions of this document may be illegible in electronic image products. Images are produced from the best available original document. 
Three dimensional imaging of DNA fragments during electrophoresis using a confocal detector

Laurence R. Brewer, Courtney Davidson, Joe Balch and Anthony Carrano

Human Genome Center, Lawrence Livermore National Laboratory, Livermore CA 94550

\begin{abstract}
We have measured the three dimensional distribution of DNA fragments within an electrophoretic band. The measurements were made using a confocal microscope and a photon counting photomultiplier detector. A DNA sequencing standard was loaded into glass microchannel plates containing polyacrylamide gel. The measurements were made by scanning the plates in three dimensions using a mechanical stage under computer control, while electrophoresis was taking place. We found that the distribution of DNA was the same for all the bands measured in the sequencing ladder with an approximate Gaussian distribution along all three axis. These measurements are important to understand what physical forces shape electrophoretic bands confined by a channel and also as an aid in the design of high throughput DNA sequencers.
\end{abstract}

\title{
1. EXPERIMENTALAPPARATUS
}

\subsection{Microchannel plates}

The microchannel plates and spacer are shown in Fig. 1. These components were clamped together to form a sandwich that was filled with a 6 percent polyacrylamide gel. The bottom plate contained three microchannels that were each $1.25 \mathrm{~mm}$ wide by 0.2 $\mathrm{mm}$ deep. The mylar spacer was 50 micron thick. The microchannels were fabricated by either ultrasonic milling or chemical etching. The loading wells were formed by the meniscus of the gel in each microchannel. A potential of 2500 volts was applied to the plates resulting in an electric field of $65 \mathrm{~V} / \mathrm{cm}$ running parallel to the microchannels. Laser excited fluorescence from the dye tagged DNA was detected approximately $25 \mathrm{~cm}$ from the load wells. Also shown 
in Fig. 1 is the orientation of the $X, Y$, and $Z$ mechanical stage scanning axis with respect to the microchannel plate.

\subsection{Confocal microscope detector}

A confocal microscope detector (1) was used to measure the spatial distribution of DNA in electrophoretic bands because of its high spatial resolution. A schematic of the optics used in the experiment is shown in Fig. 2. An argon-ion laser was run at a wavelength of $488 \mathrm{~nm}$. The laser beam passed through a 50 percent beam splitter so that a helium-neon (HeNe) laser could be superimposed on it. This was particularly useful for aligning the fluorescence collection optics. The argon-ion laser beam then entered the lamp port of a Zeiss Axioplan microscope (the lamp optics had been removed), and was reflected by a dichroic beamsplitter through a Zeiss 32 magnification, 0.4 numerical aperture, infinite conjugate objective. The full width half maximum of the intensity profile of the focused beam in air was measured to be 1.8(2) microns along both vertical and horizontal axis. These values were certainly larger in the polyacrylamide gel. The power of the argon-ion laser beam emerging from the microscope objective was approximately $0.5 \mathrm{~mW}$.

The fluorescence from the dye tagged DNA was collected by the same microscope objective, passed back through the dichroic beam splitter, through a $488 \mathrm{~nm}$ blocking filter (for eye safety), through the microscope tube lens, which focused the light through a $.64 \mathrm{~mm}$ pinhole mounted on a precision $X Y Z$ translation stage. The addition of the pinhole narrowed the depth of focus of the microscope.

The fluorescence then passed through a focusing lens, two $10 \mathrm{~nm}$ wide (FWHM) bandpass filters centered at $540 \mathrm{~nm}$ (corresponding to the "FAM" dye label in $A B I T A Q$ ) before finally impinging on the photocathode of a cooled Hammamatsu 943-02 photomultiplier tube.

The HeNe laser was used to align the collection optics as follows. A white card was placed at the focus of the beam. The scattered red light was collected by the microscope collection optics and produced a spot visible at the pinhole. The spot could be

1) Xiahua C. Huang, Mark A. Quesada and Richard A. Mathies Anal Chem,64, 967-972 (1992) "Capillary array electrophoresis using laser-excited confocal fluorescence detection." 
used to align the position of the pinhole and the photomultiplier tube. The axial depth of focus of the confocal microscope detector was measured by placing a mirror at the focus of the HeNe laser beam. A small amount of this light was detected by the photomultiplier tube and by changing the axial position of the mirror with respect to the microscope, the depth of focus was measured. This measurement is shown in Fig. 3. The full width half maximum, adjusted for light with a wavelength of $540 \mathrm{~nm}$ was $33(3)$ microns in air. Once again, this value was certainly larger in the polyacrylamide gel.

\subsection{Electronics and data acquisition}

A photon counting detection system was used. The signal from the photomultiplier tube was sent to a preamplifier, a discriminator, and then a frequency counter. The signal was integrated over times ranging from .1 to 2 seconds. The frequency counter output was sent to an IBM compatible 486, DX-2 computer. The microscope $X Y$ stage and $Z$ axis focus controller were controlled by computer via a GPIB interface. The electrophoresis plates were moved in discrete steps by the $X Y$ stage and focus controller, and data was taken while the plates were stationary, in between steps.

\section{EXPERIMENT AND DATA}

A DNA sequencing ladder (TAQ) was loaded into the microchannel plate loading wells in a vertical orientation. A potential of 2500 Volts was applied to the plates for 10 minutes and the plates were then held by the mechanical stage under the confocal microscope detector in a horizontal position. The fluorescence signal from the DNA was monitored while electrophoresis was taking place. When an electrophoretic band of interest was observed, a key on the computer keyboard was struck and the plates were mechanically scanned. A graph of the monitored fluorescence signal when the plates were stationary is shown in Fig. 4 . This data gave information equivalent to a scan parallel to the microchannel ( $X$ axis), so scans were taken in the plane perpendicular to this axis. The scans were generally taken rapidly enough so that the electropheretic bands appeared to be stationary to the confocal detector.

Figure 5 shows a scan taken along the $Y$ axis across the three microchannels. The confocal microscope detector was positioned 25 
$\mathrm{cm}$ from the channel load wells and approximately 200 microns from the bottom of the channels. The microscope $X Y$ stage moved the plates back and forth across the three channels and data was taken along each pass. DNA was only loaded into the middle channel. The data starts with a band with a length of 221 nucleotides. It took approximately 5 seconds for each scan across the 3 channels. The data shown is raw data, with no signal processing. The rectangular step seen across the three channels corresponds to the fluorescence of the gel (which is greater than that of the glass) in the microchannel. The DNA is seen in the middle channel on top of the pedestal due to the fluorescence of the gel. The DNA was highest in intensity in the middle of the channel and confined from the walls of the channel. During the experiment we looked at DNA fragments with length ranging from 50 to 300 nucleotides and all these bands showed an identical distribution of DNA in the microchannel.

Figures 6,7 , and 8 show a two dimensional scan of an electrophoretic band of DNA 56 nucleotides long. The scan was in the $Y Z$ plane perpendicular to the channel axis. The scan was taken by starting approximately 220 microns above the bottom of the channel, scanning across the channel, moving 20 microns deeper into the channel, scanning back across the channel, and continuing this cycle for 10 scans. 25 data points were taken across the channel and this produced a 250 point grid of the distribution of DNA at the peak of the electropheretic band. Fig. 6 shows the result of such a measurement. Fig. 7 shows the identical measurement taken when there was no DNA present at that position in the channel. The signal was only due to the fluorescence from the glass and gel. Notice that the profile of the signal across the channel is rectangular (apart from some spikes due to dirt or dust which are also visible in Fig. 6) and not peaked in the middle of the channel as in Fig. 6. Subtraction of this baseline data in Fig. 7 from the data in Fig. 6 results in a graph where the signal is only due to fluorescence from the dye tagged DNA. This data is presented in Fig. 8. Once again the DNA distribution is peaked in the middle of the channel and confined from the walls of the channel.

Fig. 9 shows a scan along the $Z$ axis of the microchannel through a band of DNA fragments 56 nucleotides long. This scan allowed us to determine if the DNA was confined by the glass walls formed by the top and bottom plate of the microchannel as it was by the side walls of the microchannel. The DNA distribution along the $Z$ 
axis of the band was observed to be the same as along the $Y$ axis. A Gaussian fit of the DNA distribution is also shown.

\section{DISCUSSION AND CONCLUSIONS}

We have measured the spatial distribution of DNA fragments in electrophoretic bands in a sequencing ladder that were confined by a microchannel. The DNA was measured to be peaked in intensity in the middle of the channel and confined away from the walls with an approximate Gaussian distribution. A number of physical mechanisms may be responsible for repelling the DNA from the microchannel walls such as the electrostatic force due to the field distribution in the channel, or some interaction between the DNA mobility and the thermal distribution in the microchannel. The DNA distribution could also be due to the shape of the loading well. Detailed modeling calculations will have to be made, accounting for these mechanisms before an accurate understanding of these observations can be made. It would be particularly interesting to compare the distribution of DNA for a band in a slab and a band in a microchannel that were loaded under identical conditions. Another interesting extension of this work would be to take rapid, two dimensional scans perpendicular to the channel axis. In this way a movie of the electrophoretic bands could be made as they marched down the microchannel. In the future similar spatial measurements may be attempted on electrophoretic bands in capillaries. This work was performed by Lawrence Livermore National Laboratory under the auspices of the U.S. Department of Energy Contract No. W-7405-Eng48. 


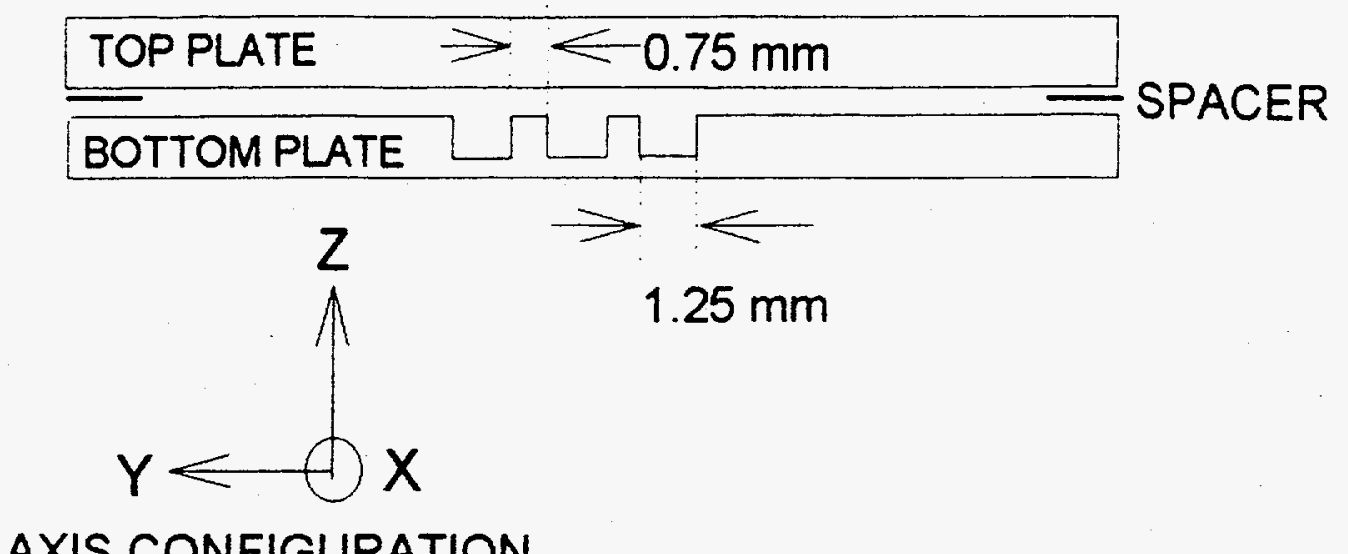

Fig. 1 Cross sectional view of the microchannel plate system. The channel depth, along the $Z$ axis is $200 \mu \mathrm{m}$. The spacer thickness is $50 \mu \mathrm{m}$.

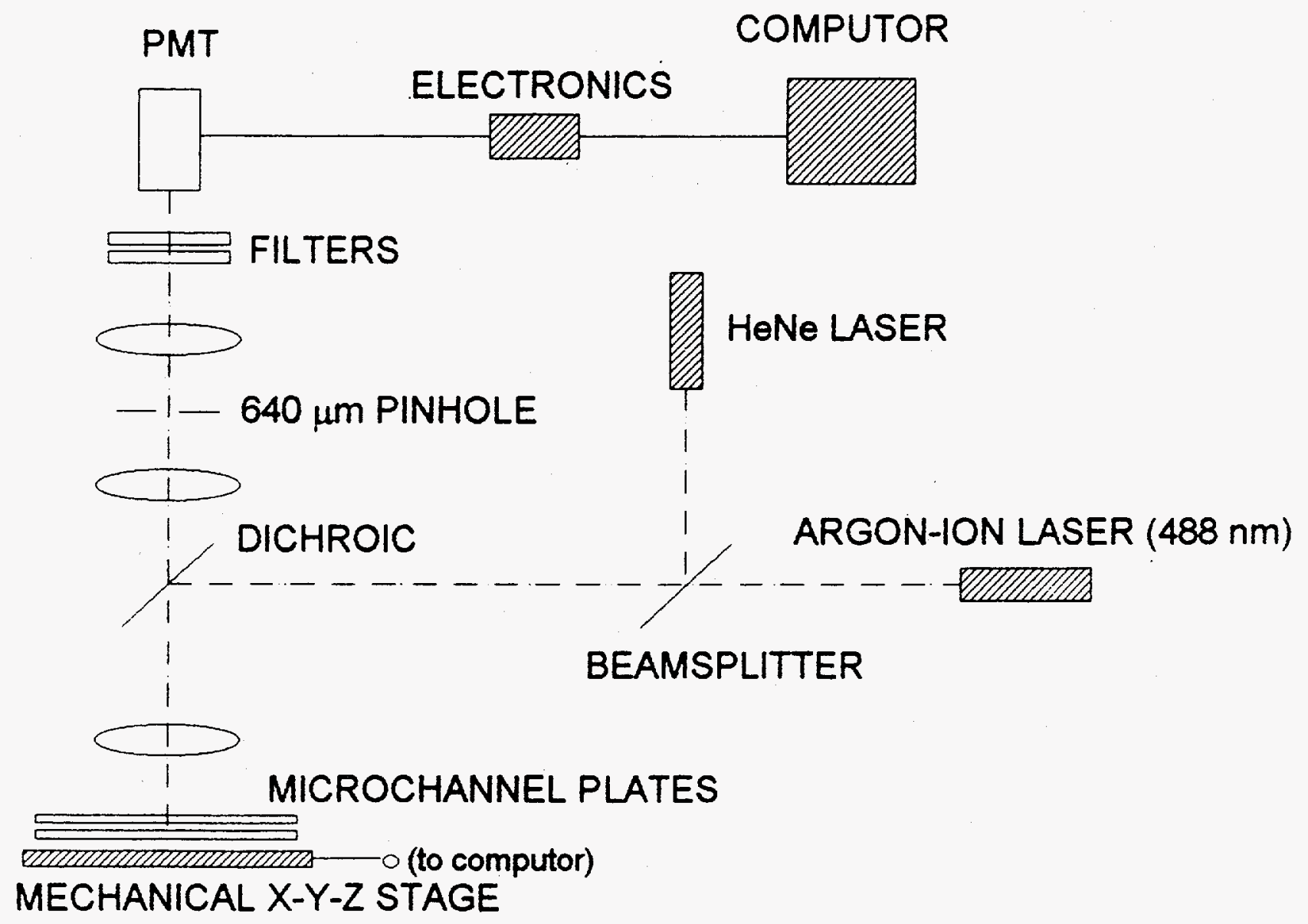

Fig. 2 The optical system for the confocal microscope detector. 


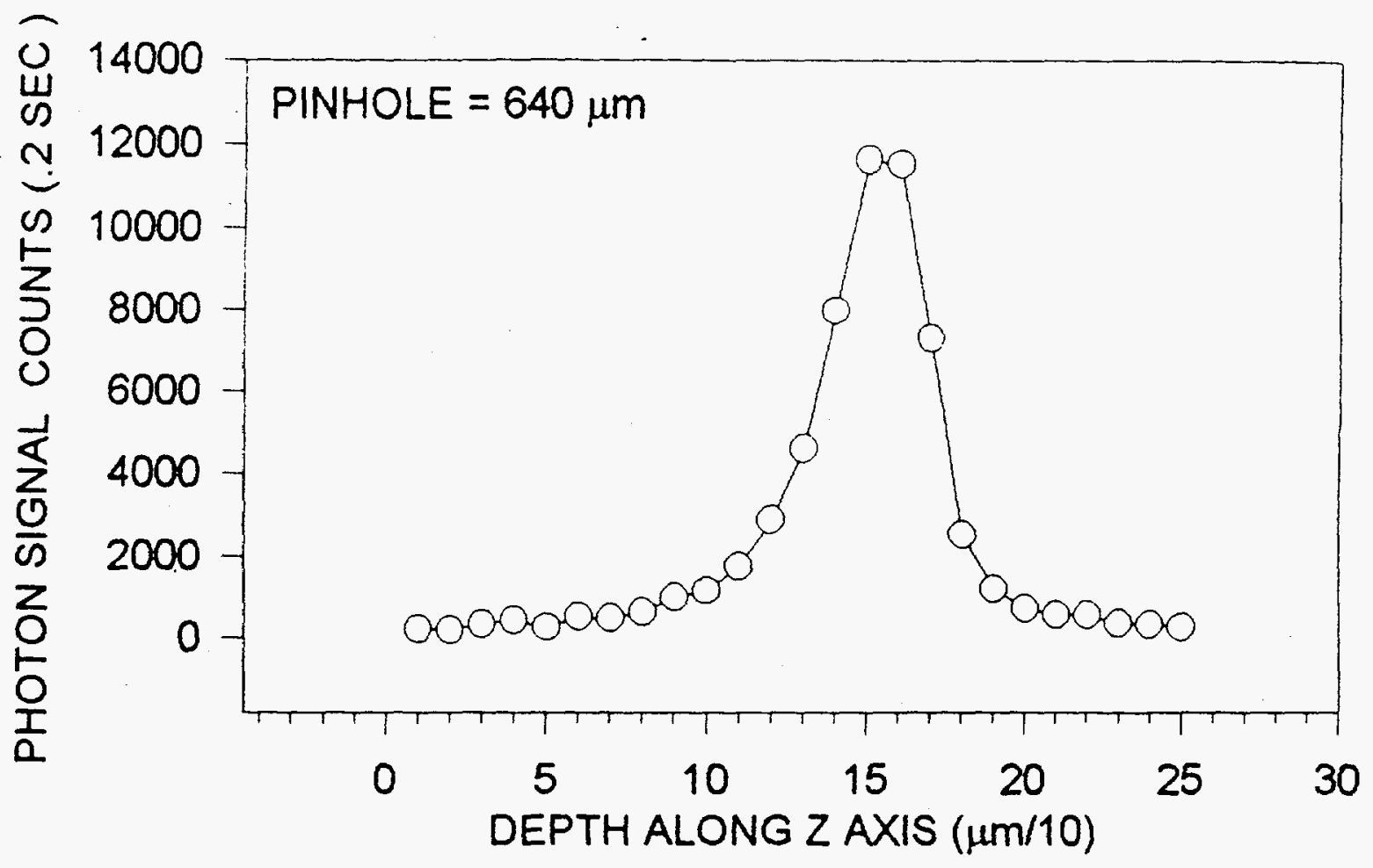

Fig. 3 The confocal microscope detector depth of focus $=$ $33(3) \mu \mathrm{m}$ (FWHM) at $\lambda=540 \mathrm{~nm}$.

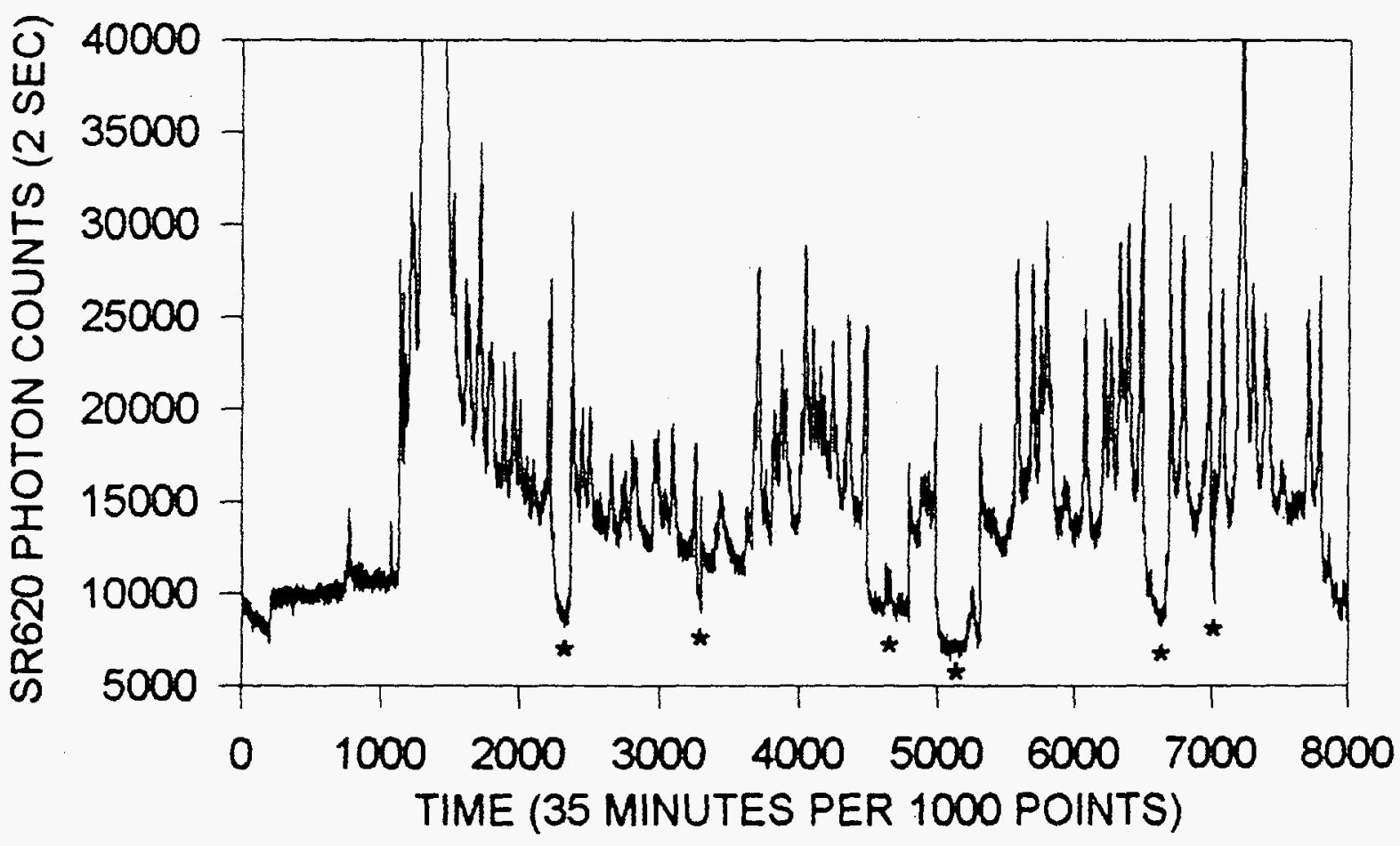

Fig. 4 The fluorescence signal from Taq as it is electrophoresed in the middle of three microchannels. The asterisks indicate places where mechanical scans occured. The data presented extends from roughly 50 to 300 nucleotides. 


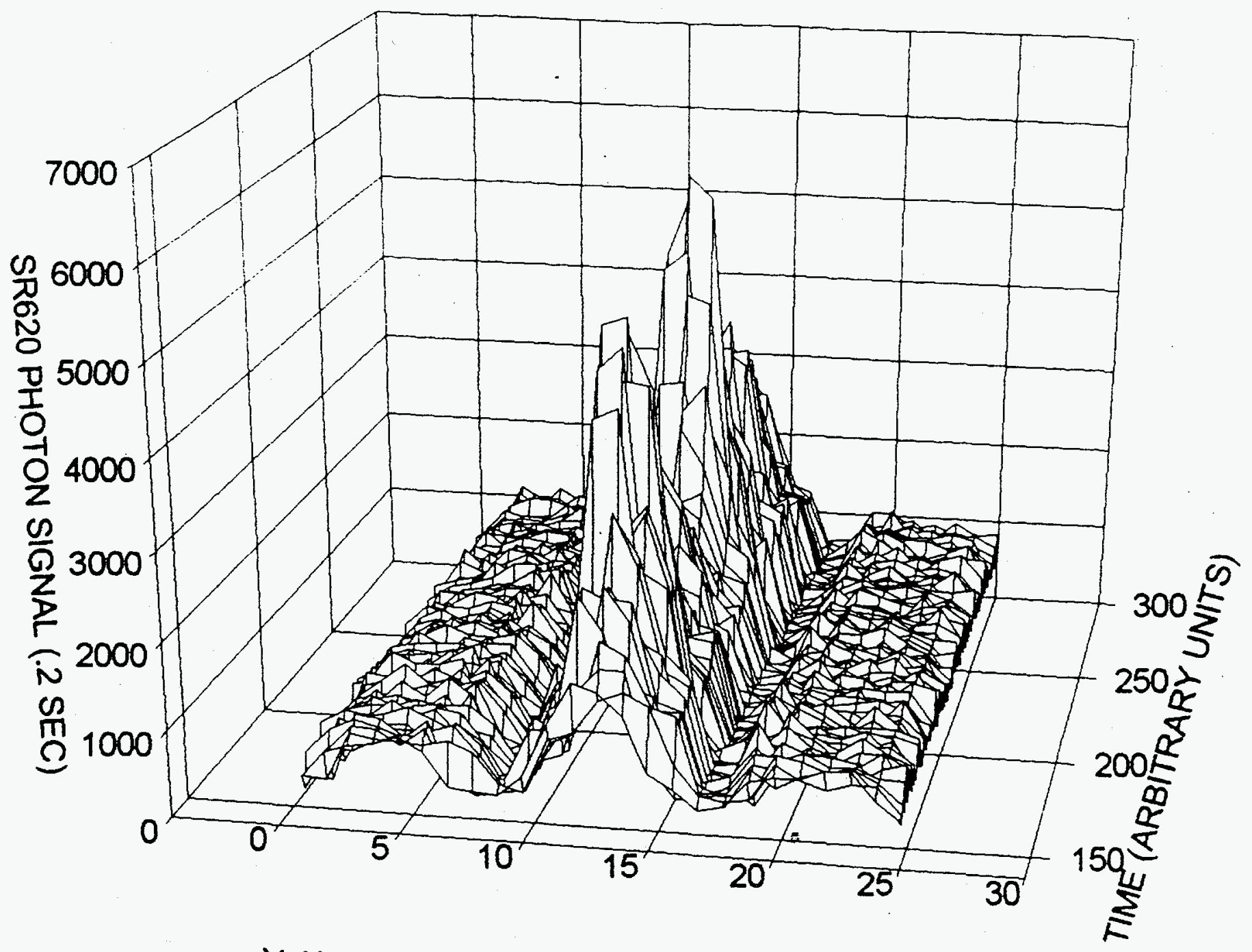

Y AXIS (PERPENDICULAR TO CHANNELS)

Fig. $5 Y$ axis scan across the three microchannels while electrophoresis was taking place. Taq sequencing standard was loaded only into the middle channel. The step seen in the outside channels is due to the fluorescence of the gel and the glass. The fragments start with a length of 221 nucleotides. 


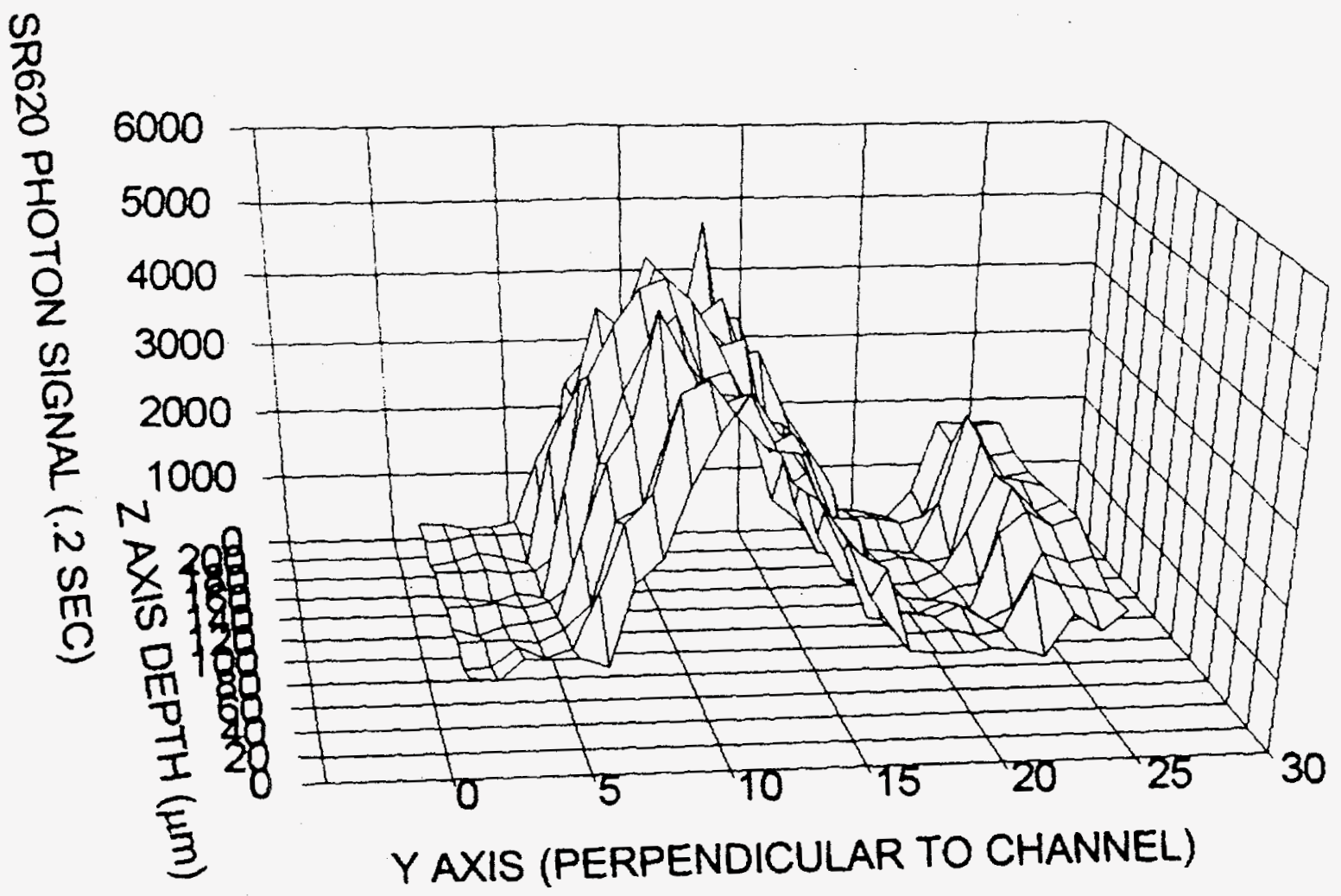

Fig. 6 The distribution of DNA in an electrophoretic band (56 nucleotides) in the $Y Z$ plane of the middle microchannel before background subtraction.

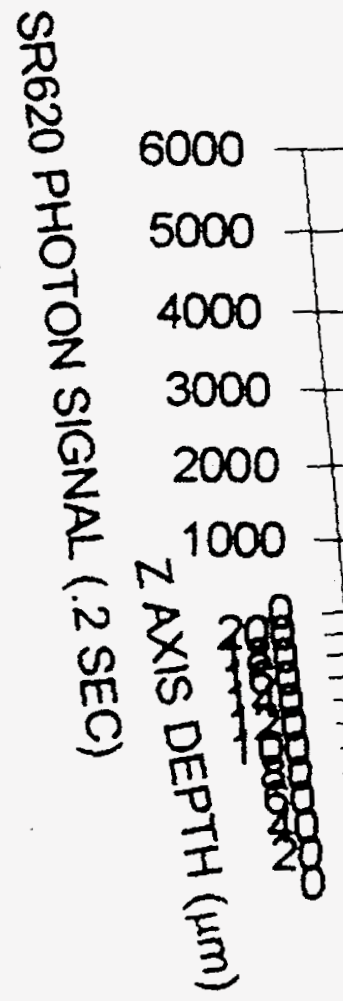

Fig. 7 The background fluorescence from the microchannel gel and glass. 


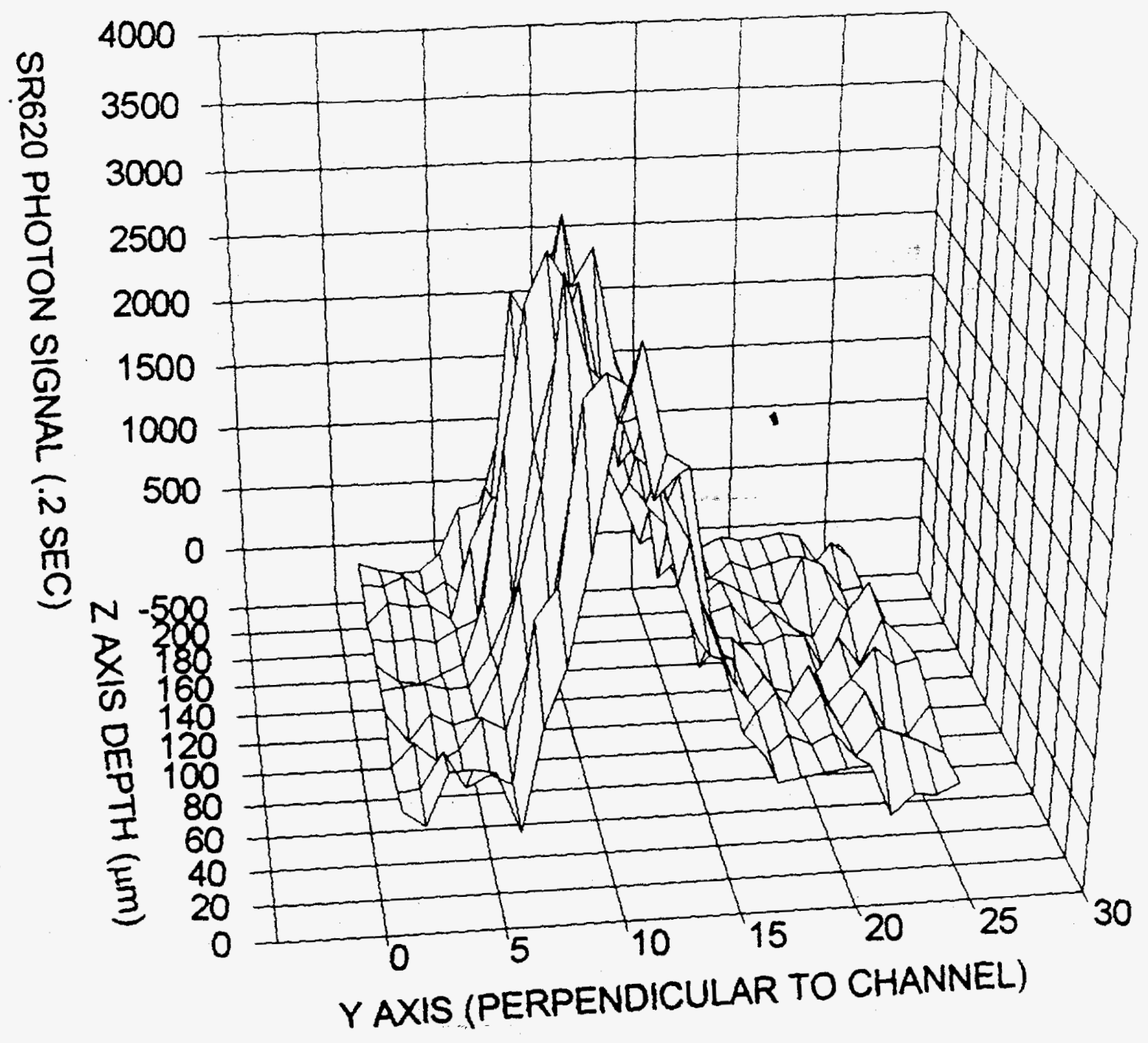

Fig. 8 The resultant DNA distribution after Fig. 6 has been subtracted from Fig. 5. The fluorescent signal is only due to DNA. 


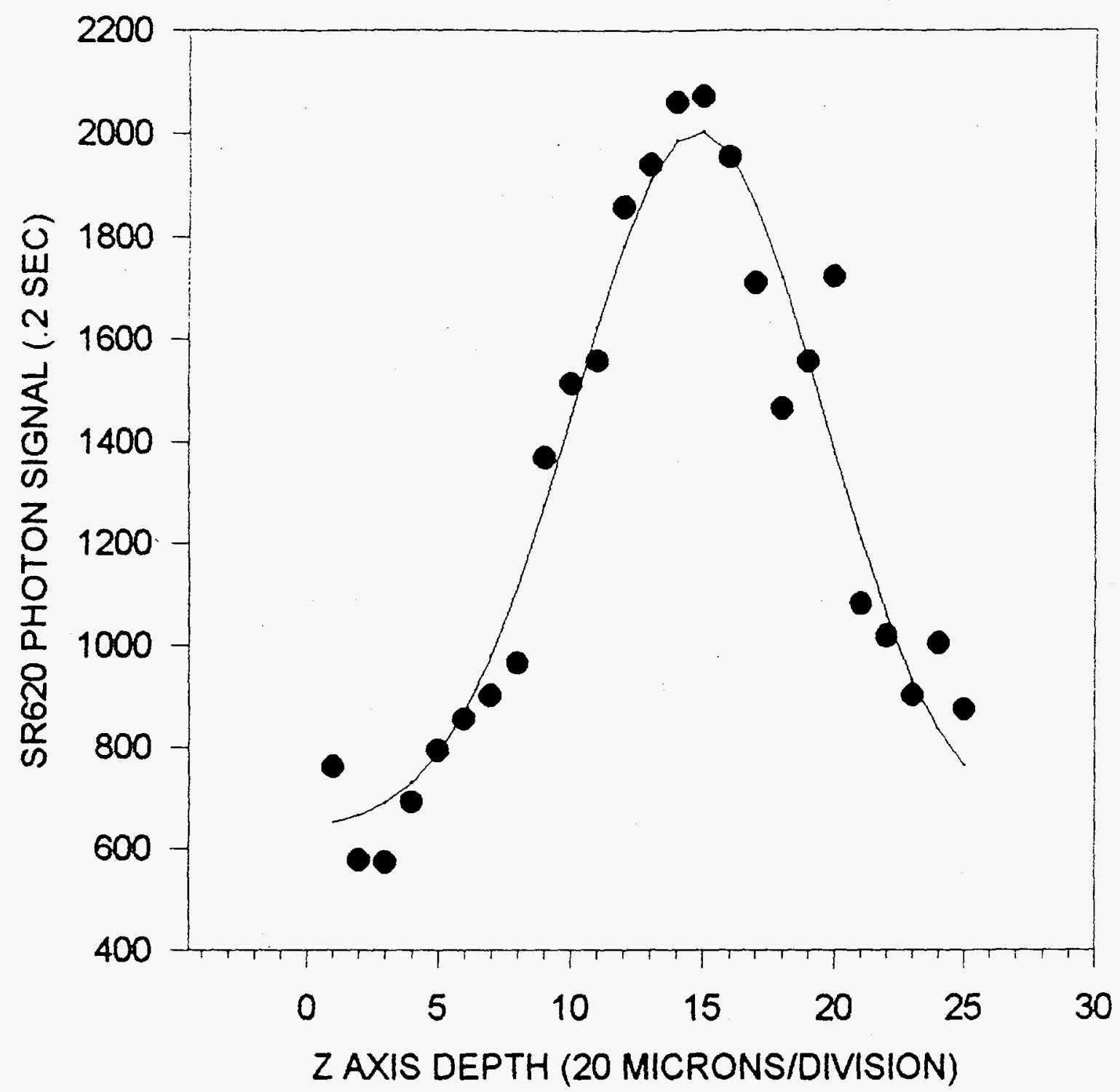

Fig. 9 The DNA distribution along the $Z$ axis of an electrophoretic band ( 56 nucleotides). The distribution fits a Gaussian profile well. 